\title{
Nuclear localization of the Arabidopsis APETALA3 and PISTILLATA homeotic gene products depends on their simultaneous expression
}

\author{
Brian McGonigle, ${ }^{1,2}$ Karim Bouhidel, ${ }^{1,3}$ and Vivian F. Irish ${ }^{4}$ \\ Department of Biology, Osborn Memorial Laboratories, Yale University, New Haven, Connecticut 06520-8104 USA
}

\begin{abstract}
The Arabidopsis APETALA3 (AP3) and PISTILLATA (PI) proteins are thought to act as transcription factors and are required for specifying floral organ identities. To define the nuclear localization signals within these proteins, we generated translational fusions of the coding regions of AP3 and PI to the bacterial uidA gene that encodes $\beta$-glucuronidase (GUS). Transient transformation assays of either the AP3-GUS or PI-GUS fusion protein alone resulted in cytoplasmic localization of GUS activity. However, coexpression of AP3-GUS with PI, or PI-GUS with AP3, resulted in nuclear localization of GUS activity. Stable transformation with these fusion proteins in Arabidopsis showed similar results. The nuclear colocalization signals in AP3 and PI were mapped to the amino-terminal regions of each protein. These observations suggest that the interaction of the AP3 and PI gene products results in the formation of a protein complex that generates or exposes a colocalization signal required to translocate the resulting complex into the nucleus. The colocalization phenomenon that we have described represents a novel mechanism to coordinate the functions of transcription factors within the nucleus.
\end{abstract}

[Key Words: Arabidopsis; APETALA3; PISTILLATA; nuclear localization signals; transcription factors]

Received April 16, 1996; revised version accepted June 12, 1996.

The Arabidopsis flower is organized into four concentric whorls of organs, from outer to inner, consisting of sepals, petals, stamens, and carpels. The elaboration of this floral pattern depends in part on the action of a number of homeotic genes (for review, see Weigel and Meyerowitz 1994). The homeotic APETALA3 (AP3) and PISTIL$L A T A(P I)$ genes are required to specify petal and stamen identities in the flower (Bowman et al. 1989, 1991). Mutations in either of these genes result in similar phenotypes, consisting of homcotic transformations of petals into sepalloid structures and stamens into carpelloid structures (Bowman et al. 1989, 1991; Hill and Lord 1989|. The AP3 and PI genes have been cloned, and share homology with homeotic genes from Antirrhinum, and with several yeast and mammalian genes encoding transcription factors (Jack et al. 1992; Goto and Meyerowitz 1994). The 56 amino acid region of homology has been termed the MADS box and corresponds to the DNAbinding domains of the yeast and mammalian gene products, suggesting that these plant genes also encode transcription factors (Sommer et al. 1990). In addition to the DNA-binding domain, the plant MADS box genes share

\footnotetext{
${ }^{1}$ These authors contributed equally to this work. Present addresses: ${ }^{2}$ DuPont Central Research, Wilmington, Delaware 19880-0402 USA; ${ }^{3} 57100$ Thionville, France.

${ }^{4}$ Corresponding author.
}

a conserved motif called the $\mathrm{K}$ box, which may be required for protein-protein interactions (Ma et al. 1991; Pnueli et al. 1991).

Expression of $A P 3$ and $P I$ together are sufficient to specify petal and stamen identities in the flower. The $A P 3$ and $P I$ genes are initially expressed in second and third whorl floral organ primordia, and expression is maintained in these regions during the differentiation of petals and stamens (Jack et al. 1992; Goto and Meyerowitz 1994). PI transcripts are also found in fourth whorl primordia at an early stage in development, although this domain of expression is not maintained /Goto and Meyerowitz 1994). Ectopic expression of both of these genes in the first whorl is sufficient to confer petal identity (Krizek and Meyerowitz 1996). Ectopic expression of AP3 in the fourth whorl where PI is normally expressed results in stamen development (Jack et al. 1994). Furthermore, the maintenance of $A P 3$ and $P I$ transcription is dependent on the expression of both $A P 3$ and $P I$ gene products (Goto and Meyerowitz 1994; Jack et al. 1994). Deficiens $(D E F)$ and Globosa (GLO), the AP3 and PI homologs in Antirrhinum, have been shown to encode proteins that bind to DNA as a heteromultimeric complex in vitro (Sommer et al. 1990; Trobner et al. 1992; Zachgo et al. 1995). Together, these observations suggest that the AP3 and PI proteins may interact to form a transcrip- 
tion factor complex that binds to regulatory sequences within the AP3 and PI promoters, resulting in auto- and cross-regulatory activation.

To test whether AP3 and PI protein distribution is regulated at the subcellular level, we have assayed the ability of fusion proteins containing these sequences to be targeted to the nucleus. Appropriate subcellular localization of nuclear-acting transcription factors is an important regulatory mechanism for many morphogenetic processes. For instance, spatially regulated nuclear localization of the Drosophila Dorsal protein dictates the development of the dorsal-ventral pattern (Roth et al. 1989; Rushlow et al. 1989; Steward 1989|. In plants, nuclear localization of some proteins involved in mediating photomorphogenesis appears to be light-regulated (Harter et al. 1994; von Arnim and Deng 1994). Proteins are targeted to the nucleus via nuclear localization signals (NLSs), which are recognized by the nuclear pore complex and actively transported into the nucleus (Richardson et al. 1988; Garcia-Bustos et al. 1991). NLS sequences have been identified in proteins from a variety of animal and plant species and can confer nuclear localization when fused to non-nuclear proteins (Garcia-Bustos et al. 1991; Raikhel 1992). The sequences of NLS motifs can be quite variable, although all tend to contain a region of basic amino acids (Dingwall and Laskey 1991).

To define the NLSs within the AP3 and PI proteins, we generated translational fusions of the coding regions to the bacterial uidA gene, which encodes $\beta$-glucuronidase (GUS), and followed the subcellular localization of GUS enzymatic activity. Although both the AP3 and PI coding regions contain multiple NLS consensus sequences, we have found that neither the AP3 nor PI fusion protein can be localized to the nucleus independently. Based on results suggesting that AP3 and PI proteins bind to each other in vitro (Goto and Meyerowitz 1994), we reasoned that these proteins may have to interact to be efficiently targeted to the nucleus. We have found that coexpression of AP3 and PI appears to be a necessary prerequisite for the efficient localization of these proteins to the nucleus. These nuclear colocalization signals map to the amino termini of the AP3 and PI proteins. These results indicate that the AP3 and PI homeotic gene products coordinate their functions in specifying floral organ identities in part by utilizing a novel nuclear colocalization mechanism.

\section{Results \\ Coexpression of AP3 and PI is required for nuclear targeting}

Because AP3 and PI are believed to act as transcription factors, we thought it likely that these proteins would contain NLS sequences. An examination of the amino acid sequences of each of these proteins indicates that each contains regions of basic amino acids that resemble NLS consensus sequences (Fig. 1A) (Dingwall and Laskey
A
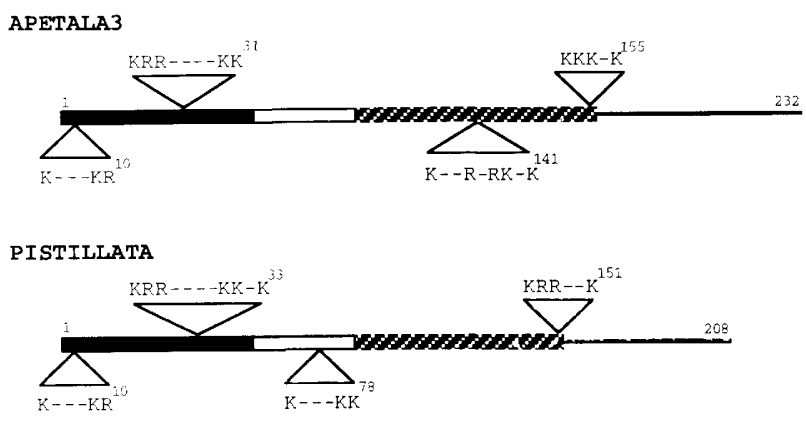

B

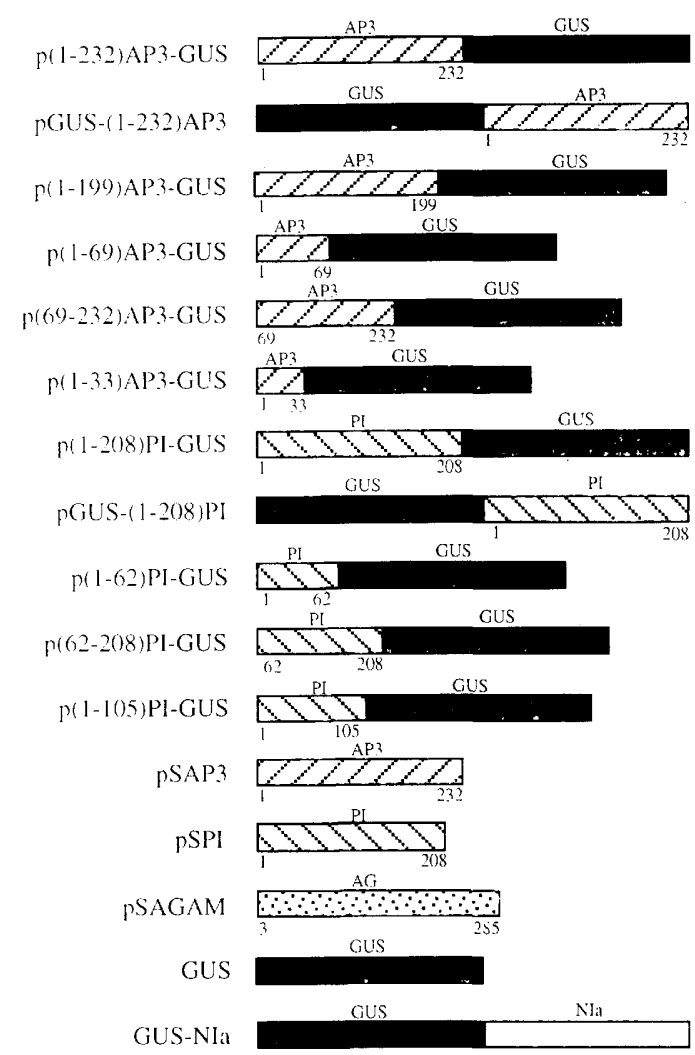

Figure 1. Constructs used in this study. $(A)$ Diagrams of AP3 and PI amino acid sequences (Jack et al. 1992; Goto and Meyerowitz 1994) with potential NLS sequences indicated. The locations of the MADS box domains are indicated by a thick black bar, I boxes by an open bar, and $\mathrm{K}$ boxes by a hatched bar (Jack et al. 1992; Goto and Meyerowitz 1994; Huang et al. 1996). (B) Diagrams of constructs used in this study. All coding regions were expressed under the control of the cauliflower mosaic virus $35 \mathrm{~S}$ promoter and the nopaline synthase terminator. The amino acids used in the fusions are indicated below each construct. In those cases in which internal fragments were used in fusions, an in-frame ATG was introduced immediately 5 ' to the fragment. Because the AG cDNA does not have an initiator ATG (Yanofsky et al. 1990), an ATG was introduced at codon 3.

1991; Garcia-Bustos et al. 1991; Raikhel 1992). Because the structure of bipartite NLS sequences consists of two 
basic domains separated by a spacer region of variable length, the basic domains that we have defined in the AP3 and PI proteins may act independently, or in concert with other basic domains.

To test the ability of these sequences to confer nuclear localization, we generated a number of constructs. These contained either AP3 or PI coding sequences fused to the uid $A$ sequence encoding GUS, driven by the constitutive cauliflower mosaic virus $35 \mathrm{~S}$ promoter (Fig. 1B). The constructs were introduced into Allium cepa (onion) epidermal cells using microprojectile bombardment |Klein et al. 1987; Varagona et al. 1992). Subcellular localization of GUS enzyme activity was assayed after $24 \mathrm{hr}$, and nuclei were detected by staining for DNA with $4^{\prime}, 6$ - diamido-2-phenylindole (DAPI). In each set of experiments, we assayed a control construct encoding the 68 -kD GUS protein, which has been shown previously to be localized to the cytoplasm (Restropo et al. 1990; van der Krol and Chua 1991). In addition, we also tested a nuclear-localized control consisting of GUS fused to the plant potyviral NIa sequence, which contains a strong bipartite NLS (Carrington et al. 1991). Cells were scored as showing nuclear localization of GUS activity if staining was seen predominantly in the nuclei with little cytoplasmic staining or if staining was seen in approximately equivalent levels in the nuclei and cytoplasm. Cells were scored as cytoplasmic if there was exclusion of GUS staining from the nucleus when optically focusing through the cell.

Expression of a full-length AP3-GUS fusion $[\mathrm{p} / 1-$ 232|AP3-GUS, Fig. 1B] in onion cells resulted in predominantly cytoplasmic GUS staining (Fig. 2C). Similarly, expression of the PI-GUS fusion [p(1-208)|PI-GUS, Fig. 1B] also showed cytoplasmic GUS staining (Fig. 2D). In both cases, the full-length AP3 and PI fusion proteins showed approximately the same basal level of nuclear staining as seen with the GUS protein alone (Fig. 3). Cotransformation of onion cells with the p(1-232)AP3GUS fusion in combination with the pSPI plasmid /containing the PI coding sequence) resulted in a high proportion of cells showing nuclear localization of GUS enzyme activity (Figs. 2E and 3). Transformation of the full-length $p(1-208)$ PI-GUS fusion, in combination with a plasmid containing the AP3 coding sequence (pSAP3), also resulted in high levels of nuclear GUS activity (Figs. $2 \mathrm{~F}$ and 3). Carboxy-terminal fusions of GUS-AP3 and GUS-PI were also assayed independently and in colocalization experiments and were found to act similarly to the amino-terminal fusions [pGUS-(1-232)AP3, pGUS(1-208)PI, Fig. 1B; data not shown]. However, the carboxy-terminal fusions showed reduced levels of GUS expression, and therefore we used amino-terminal fusions to GUS in all subsequent experiments.

\section{Specificity of the AP3-PI interaction}

To test whether the colocalization effect we had observed was specific, we also cotransformed onion cells with either the AP3-GUS or PI-GUS fusions in combi-

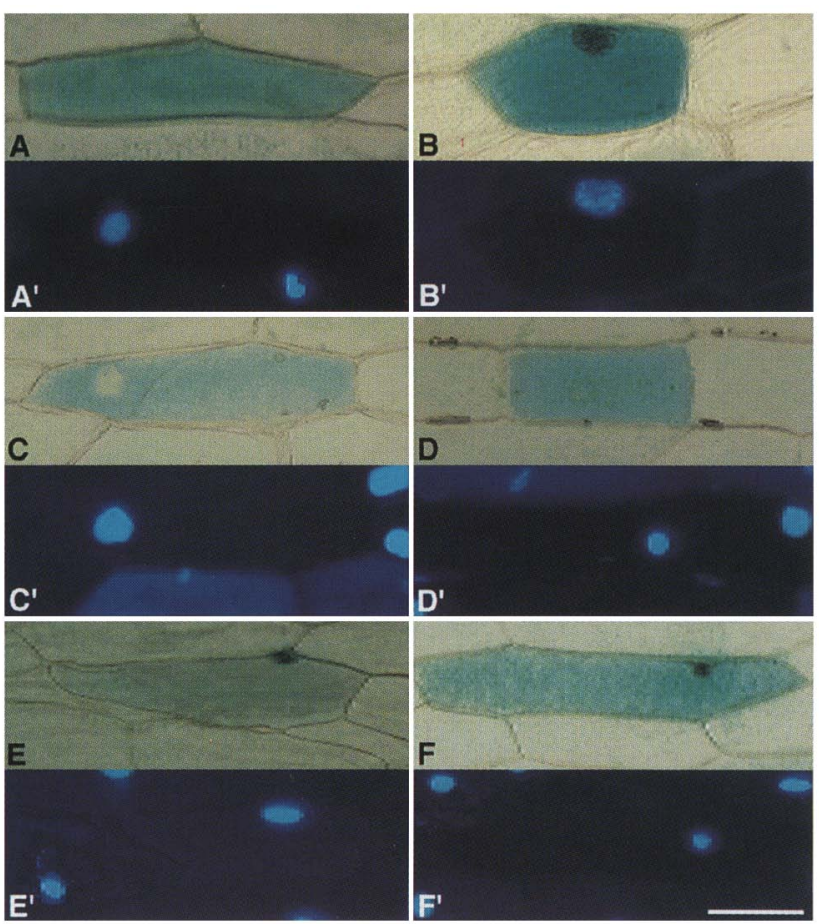

Figure 2. Subcellular localization of AP3 and PI combinations. Onion cells were transiently transformed with various constructs and stained for GUS activity. Each field of cells was photographed in bright field $\mid$ top $\mid$ to visualize GUS staining and epifluorescence (bottom) to show DAPI staining of the nuclei. $(A)$ Expression of GUS alone is cytoplasmic; $(B)$ expression of the GUS-NIa construct results in enhanced nuclear GUS activity; $(C)$ expression of $p(1-232) A P 3-G U S$ results in cytoplasmic GUS staining; $(D) \mathrm{p}(\mathrm{I}-208)$ PI-GUS expression also results in cytoplasmic GUS activity; $(E)$ transformation with $\mathrm{p}(1-$ 232)AP3-GUS in combination with pSPI results in nuclear localization of GUS activity; $(F)$ transformation of $p(1-208)$ PIGUS in combination with pSAP3 also results in nuclear localization of GUS activity. Bar, $100 \mu \mathrm{m}\left(A, A^{\prime}-F, F^{\prime}\right)$.

nation with a plasmid containing the coding region of the AGAMOUS (AG) gene, driven by the $35 \mathrm{~S}$ promoter (pSAGAM, Fig. 1B). The $A G$ homeotic gene encodes a MADS box containing protein that is required for stamen and carpel development (Bowman et al. 1989, 1991; Yanofsky et al. 1990). Cotransformation of p(1-232)AP3GUS with pSAGAM resulted in cytoplasmic localization of GUS activity, indicating that AG cannot substitute for PI sequences in nuclear colocalization of AP3 (Fig. 3). However, cotransformation of $\mathrm{p}(1-208)$ PI-GUS with pSAGAM resulted in a low level of nuclear localization of GUS activity. These results suggest that the AG protein can partially substitute for AP3 in conferring nuclear localization on the PI-GUS fusion protein.

The nuclear colocalization signals map to the amino termini of AP3 and PI

We generated a series of constructs in which different domains of the AP3 protein were translationally fused to 


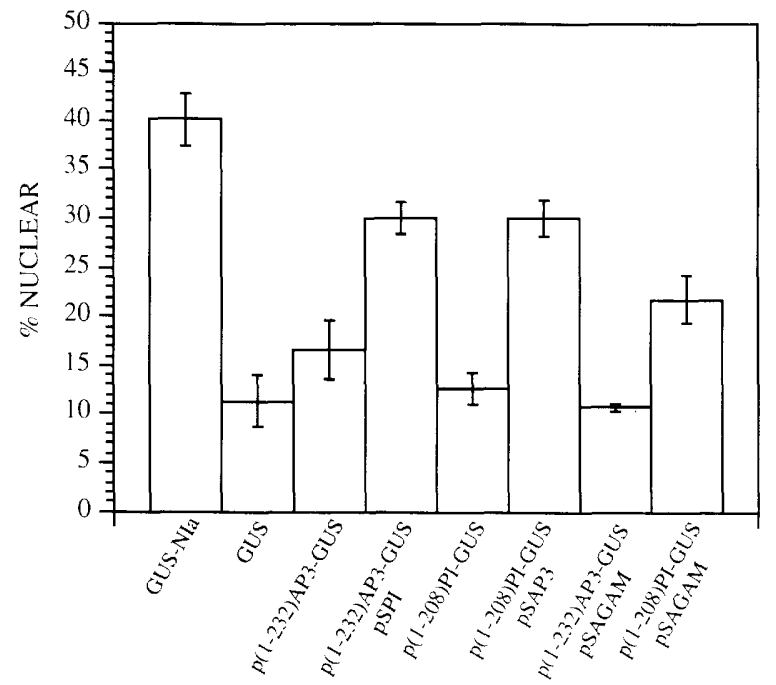

Figure 3. Specificity of nuclear colocalization. The percent of nuclear localization of GUS activity observed for various plasmids (see Fig. 1 for details of plasmid constructs) in transient assays is shown graphically. Plasmids were introduced independently or in combinations (as noted below each column) into onion cells using microprojectile bombardment, and the subcellular localization of the resulting GUS activity was scored. Each column represents a total of five independent onion cell transient assays, with a minimum of 100 GUS-expressing cells scored for each individual assay. Standard deviations are indicated.

the GUS coding sequence (Fig. 1B). These constructs were assayed for GUS expression in onion cells individually and in combination with the pSPI plasmid containing the entire PI coding sequence (Fig. 4). A truncated AP3 protein containing 199 amino acids translationally fused to GUS [p(1-199|AP3-GUS] and cobombarded with pSPI showed a high percentage of cells with nuclear staining, comparable to that seen with the full-length AP3-GUS fusion in combination with pSPI (Figs. 1B and 4). AP3 amino acids 69-232 [p(69-232)AP3-GUS] were not able to target GUS expression to the nucleus in combination with PI, indicating that the carboxy-terminal region of the AP3 protein is not sufficient for nuclear colocalization. The amino-terminal region of the AP3 protein [ $p(1-69)$ AP3-GUS] retained the ability to drive GUS expression into the nucleus in combination with the PI protein. Cells expressing p(1-69)AP3-GUS alone, however, showed background levels of nuclear GUS activity. A fusion of AP3 amino acids 1-33 to GUS showed low levels of nuclear staining in combination with the PI protein.

We also generated a series of plasmids containing various domains of the PI coding sequence translationally fused to GUS (Fig. 1B). These fusions were assayed alone and in combination with the AP3 protein (pSAP3) for subcellular localization of GUS expression in onion cells (Fig. 5). Similar to what we observed for AP3, we found that PI sequences required for nuclear colocalization

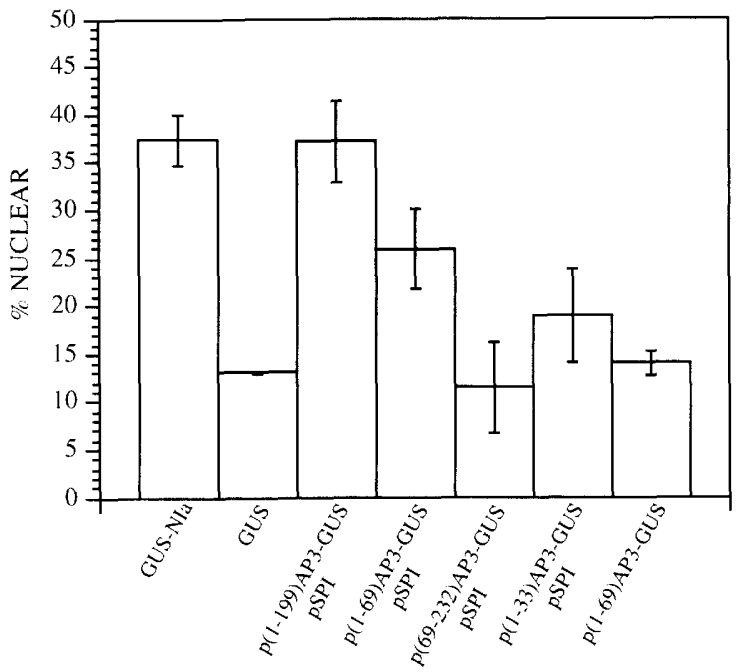

Figure 4. The region required for AP3 nuclear colocalization maps to the amino terminus. The percent of nuclear localization of GUS activity observed for various plasmids in transient assays is shown, as in Fig. 3. Each column represents a total of six independent onion cell transient assays.

map to amino-terminal amino acids $1-105$. When fused to GUS and expressed in combination with pSAP3, PI amino acids 1-62 showed weak levels of nuclear colocalization. The carboxy-terminal region of PI $[\mathrm{p} / 62-$ 208|PI-GUS] was not sufficient for nuclear colocalization. The attenuated ability of increasingly shorter amino-terminal domains of the PI protein to localize GUS expression to the nucleus in combination with AP3 suggests that there may be several sequences in the amino-terminal region of the PI protein that participate in nuclear colocalization.

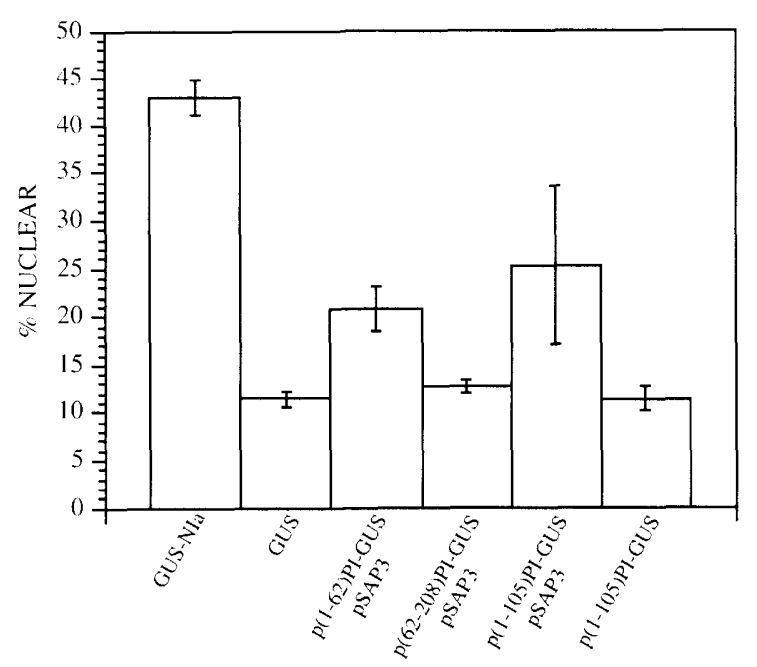

Figure 5. The region required for PI nuclear colocalization maps to the amino terminus. The percent of nuclear localization of GUS activity observed for various plasmids in transient assays is shown, as in Fig. 3. Each column represents a total of four independent onion cell transient assays. 
Stable ectopic expression of AP3 or PI results

in floral homeotic transformations

and developmentally regulated nuclear localization

To ascertain whether nuclear colocalization occurs in vivo and whether it is developmentally regulated, we generated transgenic Arabidopsis plants expressing either the p(1-232)AP3-GUS, $\mathrm{p}(1-199)$ AP3-GUS, or $\mathrm{p}(1-$ 208|PI-GUS constructs. Over 40 transgenic lines for each construct were obtained. The phenotypes produced by these plants were compared to those of transgenic Arabidopsis plants expressing either the GUS or GUSNIa constructs (Carrington et al. 1991; von Arnim and Deng 1994).

Most of the $40 \mathrm{p}(1-208)$ PI-GUS transgenic lines generated showed a mild homeotic transformation of sepal into petalloid tissue (Fig. 6A,B). The petalloid tissue was generally found at the lateral edges of the sepal, and these cells could be distinguished by their white color, as well as by their distinctive epidermal cell morphology. This phenotype is similar to that reported for ectopic expression of the PI protein (Krizek and Meyerowitz 1996). These observations indicate that the PI-GUS fusion protein functions in a manner similar to the native PI protein. No obvious phenotypes were observed in other tissues of the transgenic plants.

These p(1-208)PI-GUS transgenic lines were analyzed at the histological level, and most showed similar patterns of GUS staining in the flowers and weak staining in a number of other tissues. $\mathrm{p}(1-208)$ PI-GUS transgenic seedlings showed low but detectable levels of GUS activity in hypocotyls, cotyledons, and in the vascular tissue of the root (Fig. 6D). The overall pattern of seedling staining was, however, different from that observed for transgenic seedlings containing either the GUS or the GUS-NIa construct. In both of these cases, transgenic seedlings displayed GUS activity predominantly in the roots and cotyledon guard cells, with lower levels in the hypocotyls (Fig. 6C; data not shown). These observations suggest that post-transcriptional regulation may modulate the amount of functional p(1-208)PI-GUS gene product produced. Alternatively, the $\mathrm{p}(1-208)$ PI-GUS construct may include cryptic promoter elements mapping within the PI cDNA sequences, which modify the pattern of GUS expression and, consequently, the pattern of GUS staining observed. $\mathrm{p}(1-208)$ PI-GUS transgenic plants also displayed detectable GUS activity in young leaf primordia and in trichomes. In the flowers, the $\mathrm{p}(1-208)$ PI-GUS transgenic plants showed GUS activity predominantly in the petals and filaments of the stamens (Fig. 6G). GUS enzyme activity was also seen in a ring of tissue subtending the floral organs and in the style of the gynoecium. The p(1-208)PI-GUS expression at the base of the sepals may account for the partial homeotic transformations seen in these organs. The patterns of expression seen for this construct are in contrast to the patterns of GUS activity produced by either the GUS construct alone or by the GUS-NIa construct, where staining is seen predominantly in the sepals, stamens, and in the styles of transgenic flowers (Fig. 6F).
We investigated the subcellular localization of the $\mathrm{p}(1-208)$ PI-GUS gene product in several cell types from transgenic plants. We examined transgenic p(1-208)PIGUS hypocotyl cells, guard cells, and trichomes, and all showed cytoplasmic localization of GUS activity (Fig. $6,(N, R)$. This pattern of enzymatic activity was similar to that seen with GUS alone, whereas transgenic plants expressing GUS-NIa showed clear nuclear localization of GUS activity in these cell types (Fig. 6I,M,Q). However, in young petal cells, where AP3 is normally expressed, the $\mathrm{p}(1-208)$ PI-GUS transgenic plants displayed nuclear localization of GUS activity (Fig. 6W). This is similar to the nuclear localization of GUS activity observed in sepal cells from transgenic GUS-NIa plants (Fig. 6U).

We also generated transgenic lines containing either the $\mathrm{p}(1-232) \mathrm{AP} 3-\mathrm{GUS}$ or $\mathrm{p}(1-199)$ AP3-GUS constructs. One $p(1-199) A P 3-G U S$ transgenic line was recovered that showed a phenotype of extra stamens in the fourth whorl, similar to that shown by plants ectopically expressing the AP3 protein (Jack et al. 1994). This line was subsequently lost and was not subjected to further analysis. The other $p(1-199)$ AP3-GUS and p(1-232)AP3GUS lines obtained did not show any obvious visible phenotypes. These $p(1-232) A P 3-G U S$ and $p(1-199)$ AP3GUS lines were analyzed histologically and showed similar patterns of GUS expression to those seen for $\mathrm{p}(1-$ 208)PI-GUS in vegetative tissues (Fig. 6E; data not shown). GUS staining in the $\mathrm{p}(1-232) \mathrm{AP} 3-\mathrm{GUS}$ and $\mathrm{p}(1-$ 199|AP3-GUS flowers was limited to the petals and stamens (Fig. 6H; data not shown). At the subcellular level, we examined hypocotyl, guard, and trichome cells in transgenic plants expressing the $\mathrm{p}(1-232) \mathrm{AP} 3-\mathrm{GUS}$ construct. In these cell types, we observed cytoplasmic GUS staining (Fig. 6K,O,S). In young petal cells, where PI is normally expressed, $p(1-232)$ AP3-GUS transgenic plants showed nuclear localization of GUS activity (Fig. $6 \mathrm{XI}$.

To test whether ectopic coexpression of AP3 and PI was sufficient to confer nuclear localization in vivo, we crossed transgenic plants containing $p(1-208)$ PI-GUS with p(1-232)AP3-GUS transgenic plants. We examined the subcellular localization of GUS activity in several cell types of the progeny from these crosses. In seedlings, nuclear localization of GUS activity was seen in hypocotyl cells (Fig. 6L). Although we could not consistently detect nuclear localization of GUS staining in the cotyledon guard cells, other cotyledon cells, such as mesophyll, showed clear nuclear localization of GUS activity (Fig. 6P). In addition, we examined trichomes and observed nuclear GUS activity in these cells (Fig. 6T).

\section{Discussion}

\section{AP3 and PI cooperate in nuclear colocalization}

We have shown that the AP3 and PI proteins need to be coexpressed to be effectively localized to the nucleus. Both stable and transient expression of either the AP3 or PI fusion protein alone results in cytoplasmic localiza- 


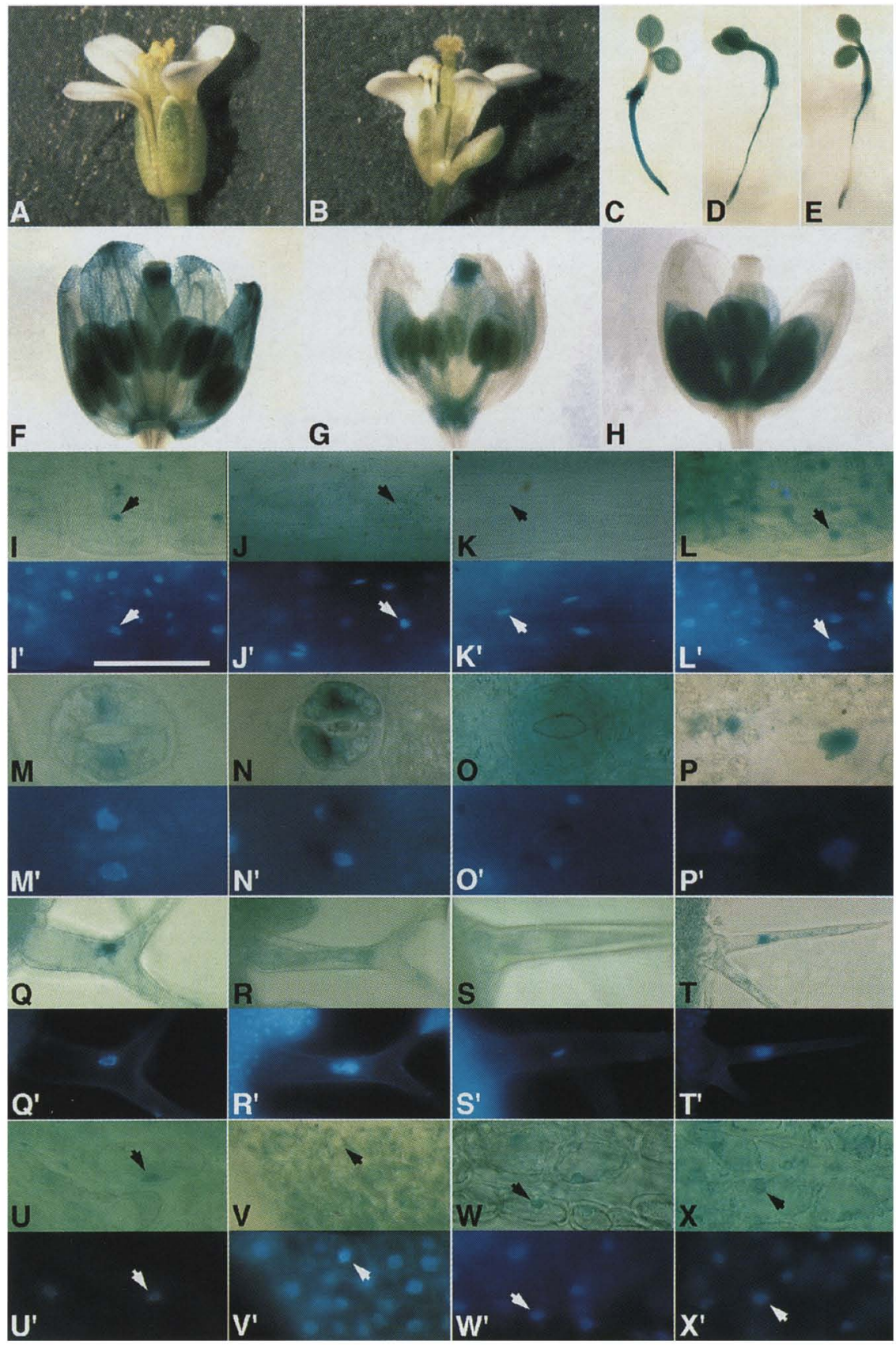

Figure 6. Expression of fusion proteins in vivo results in homeotic transformations and developmentally regulated nuclear localization. (A) The wild-type Arabidopsis flower. (B) A transgenic p(1-208)PI-GUS flower shows a mild homeotic transformation of the margins of the sepals to white petal-like tissue. $(C-H)$ Tissue distribution of GUS enzyme activity in various transgenic lines. Transgenic GUS-NIa seedlings show GUS activity predominantly in the root and cotyledons $(C)$, while $\mathrm{p}(1-208)$ PI-GUS $(D)$ and $\mathrm{p}(1-$ 232)AP3-GUS (E) seedlings show GUS activity mainly in the hypocotyls, cotyledons, and root vascular system. GUS activity in flowers from transgenic GUS-NIa plants $(F)$ is seen mainly in the sepals while GUS activity in transgenic p/1208/PI-GUS flowers $(G)$ is seen in the young petals, in the filaments of the stamens, in the style, and at the base of the floral organs. GUS activity in $\mathrm{p} / 1-$ 232)AP3-GUS flowers $(H)$ is observed in the young petals and the young filaments. In both p(1-208)PI-GUS and p(1-232)AP3GUS transgenic flowers, GUS activity appears to be restricted to early developmental stages, as it is not seen in mature flowers at anthesis. $(I-X)$ Subcellular distribution of GUS enzyme activity in various tissues of transgenic plants. Dissected floral organs were stained as described in $\mathrm{Ma}$ terials and methods and photographed in bright field (top, $I-X)$ to visualize GUS activity, and with epifluorescence (bottom, $\left.I^{\prime}-X^{\prime}\right)$ to show the position of the nuclei. Arrows indicate positions of selected nuclei. Hypocotyl cells in transgenic GUSNIa (I) seedlings show nuclear localization of GUS activity. In comparison, hypocotyl cells from $\mathrm{p}(1-208)$ PI-GUS seedlings also show cytoplasmic localization of GUS activity $(J)$, as do hypocotyl cells from $\mathrm{p}(1-$ 232)AP3-GUS seedlings (K). Seedlings containing both $\mathrm{p}(1-208) \mathrm{PI}-\mathrm{GUS}$ and $\mathrm{p}(1-$ 232)AP3-GUS constructs show nuclear localization of GUS activity in hypocotyl cells $(L)$. Cells from GUS-NIa transgenic plants also show nuclear localization of GUS activity in guard cells and trichomes $\mid M, Q)$, whereas $p(1-208) P I-G U S(N, R)$, and p $(1-232)$ AP3-GUS $|O, S|$ transgenic plants display cytoplasmic localization of GUS activity in the guard cells and trichomes. Plants containing both p(1-208)PI-GUS and $\mathrm{p}(1-232)$ AP3-GUS constructs show nuclear localization of GUS activity in mesophyll cells $(P)$, and in trichomes $(T)$. In young petal cells, p(1-208)PI-GUS $(W)$ and p(1-232)AP3-GUS $(X)$ plants display nuclear localization of GUS activity. In comparison, GUS-NIa sepal cells also show nuclear localization of GUS staining $(U)$, whereas young sepal cells from GUS transgenic lines $(V)$ show cytoplasmic staining. Bar, $100 \mu \mathrm{m}\{I-L, Q-T) ; 20 \mu \mathrm{m}(M-P, U-X)$.

tion of fusion protein activity. These assays demonstrate that neither the AP3 nor PI proteins contain functional NLS sequences. This observation is striking in that, to our knowledge, there are no known cases of nuclear colocalization of two proteins that are individually defective in nuclear transport (Dingwall and Laskey 1991).
Several models can be envisaged that could account for how this novel nuclear colocalization phenomenon is mediated. Dimerization of the AP3 and PI proteins could bring together two sequences, both of which are required for a functional NLS. Many bipartite NLSs have been identified in a variety of plant and animal proteins in 
which two discrete domains of basic amino acids are both required for nuclear localization (Dingwall and Laskey 1991; Garcia-Bustos et al. 1991; Raikhel 1992). The spacing between the two basic domains that comprise bipartite signals is variable, and experimental alteration of this spacing indicates that changing the number of intervening amino acids has little effect on those bipartite NLSs that have been tested (Dingwall and Laskey 1991; Abel and Theologis 1995). These observations suggest that it is possible that AP3 and PI could encode an intermolecular bipartite signal, as opposed to the intramolecular forms that have been described previously. Alternatively, dimerization of the AP3 and PI proteins could result in a conformational change, leading to the unmasking of an endogenous NLS present in one or both of the proteins. In this scenario, the sequences that we have defined as being required for nuclear colocalization would correspond to those sequences required for inducing the conformational change. Intramolecular masking of a NLS has been proposed as a mechanism to regulate the activity of several transcription factors. For instance, in the absence of hormone, the glucocorticoid receptor is cytoplasmically localized; upon hormone binding the glucorticoid receptor undergoes a conformational change, exposing a functional NLS (Picard and Yamamoto 1987). Similarly, p110, the precursor form of $\mathrm{NF}-\mathrm{kB}$, is retained in the cytoplasm until the molecule is cleaved, resulting in the exposure of an NLS on the mature protein (Henkel et al. 1992).

The AP3 and PI proteins may also interact within the nucleus to prevent nuclear export. Several examples of regulated nuclear export have been described recently in animal cells, and it appears that export of proteins from the nucleus can be modulated by interactions within the nucleus that cause selective retention (Laskey and Dingwall 1993; Schmidt-Zachmann et al. 1993; Fabre and Hurt 1994|. Several putative nuclear export signals have been defined, but the generality of these types of signal sequences has yet to be established (Fischer et al. 1995; Michael et al. 1995; Wen et al. 1995). It is possible that binding of an AP3-PI heterodimeric complex to DNA may be sufficient to prevent nuclear export.

\section{Sequences required for nuclear colocalization}

We have mapped the domains required for colocalization to the first 69 amino acids of AP3 and to the first 105 amino acids of PI. A shorter fusion protein containing just the first 62 amino acids of PI shows low but detectable levels of colocalization function. This observation suggests that at least two domains within the aminoterminal portion of PI, the first mapping within the first 62 amino acids, and the second between amino acids 62 and 105, may each contain sequences required for colocalization. Depending on the mechanism underlying nuclear colocalization, these domains may either contain NLS-like sequences required to form a trans-acting bipartite signal or may define domains that are required for heterodimerization and consequently for conformational changes exposing NLS sequences elsewhere in the pro- teins. Several sequences with similarity to known NLS sequences map to these regions of the AP3 and PI proteins (Fig. 1) but are apparently nonfunctional in transient and stably transformed cells. The MADS box domain from the mammalian serum response factor (SRF) protein has also been assayed for its ability to confer nuclear localization, and this domain is not sufficient for nuclear targeting (Rech et al. 1994; Gauthier-Rouviere et al. 1995). Instead, the SRF NLS sequence maps to an amino-terminal domain of the protein that is not conserved between SRF and the plant MADS box-containing gene products (Rech et al. 1994; Gauthier-Rouviere et al. 1995).

The AP3 and PI domains required for nuclear colocalization include the MADS box, plus sequences carboxyterminal to the MADS box within the region termed the I box (Fig. 1A; Huang et al. 1996). The I box has been implicated in protein-protein interactions for several MADS box-containing proteins, as deletions within the I box prevent dimerization of several Arabidopsis MADS box proteins in vitro (Huang et al. 1996). Analyses of the human SRF protein have demonstrated that sequences both within the MADS box and the 30 amino acids immediately carboxy-terminal are required for dimerization by both genetic (Norman et al. 1988) and structural criteria (Pellegrini et al. 1995). Point mutations in the analagous regions of the yeast MCMl protein have been shown to abolish or attenuate ternary complex formation (Bruhn and Sprague 1994). Furthermore, the AP3 protein can be immunoprecipitated in vitro with an epitope-tagged version of PI, providing evidence for a physical interaction between these two proteins (Goto and Meyerowitz 1994). Deletion of the first 61 amino acids of PI abolishes this binding activity (Goto and Meyerowitz 1994). These observations taken together suggest that multiple regions within these amino-terminal domains of AP3 and PI are required for dimerization.

The $\mathrm{K}$ box domain of the plant MADS box genes has also been postulated to be required for heterodimerization (Ma et al. 1991; Pnueli et al. 1991; Goto and Meyerowitz 1994). Computer modeling of the $\mathrm{K}$ boxes of these gene products predicts that these regions can form either two or three amphipathic helices (Ma et al. 1991; Pnueli et al. 1991; Jack et al. 1992; Trobner et al. 1992). Although the K box primary sequence is not highly conserved, several mutations in the plant MADS genes fall within the $\mathrm{K}$ box domain, suggesting that it is important for function (Jack et al. 1992; Schwarz-Sommer et al. 1992). Deletion of the PI K box attenuates but does not abolish the ability of an epitope-tagged version of PI to immunoprecipitate AP3 protein, suggesting that this region is not absolutely necessary for heterodimerization (Goto and Meyerowitz 1994). Our observations indicate that the $\mathrm{K}$ box is not required for nuclear colocalization; hence, heterodimerization through other parts of the proteins appears to be sufficient to confer this function.

Post-transcriptional regulation of AP3 and PI

The expression patterns and phenotypes produced by the 
AP3 and PI fusion proteins in transgenic Arabidopsis plants suggest that protein stability also plays a role in modulating their function. In transgenic p(1-232)AP3GUS and p(1-208)PI-GUS flowers, we observed high levels of GUS staining primarily in the second and third whorls, in contrast to the staining patterns seen in the GUS or GUS-NIa transgenic flowers (Fig. 6G,H). Because we do not observe high levels of GUS staining throughout the first or fourth whorls, it appears that factors required for stabilizing either GUS fusion protein are largely absent from these floral whorls. It is possible that specific destabilization factors may be operating in some first and fourth whorl tissues of the flower to degrade these AP3 and PI proteins in floral domains where they are not normally expressed.

It has been postulated that stabilization of the AP3 protein occurs via interactions with the PI protein and that protein stability is an additional aspect of AP3 and PI regulation (Jack et al. 1994). However, several lines of evidence suggest that stable AP3 or PI proteins can be detected in the absence of their heterologous partners. We have observed that AP3 and PI fusion proteins are expressed in vegetative tissues of transgenic plants, which indicates that these proteins are stable in the absence of their partner or other floral factors. Furthermore, we see GUS expression in the styles of transgenic $\mathrm{p}(1-208)$ PI-GUS flowers, where AP3 is not normally expressed. It appears that PI-GUS expression in this floral tissue is not dependent on AP3. AP3 protein is also detectable in ovules of pi-1 mutants, although the subcellular localization of the AP3 protein in this tissue has not been reported (Jack et al. 1994). In addition, we also detect GUS expression of individual fusion proteins in the heterologous onion cell system. Whereas the levels of GUS activity seen in the transient assays are likely to reflect the high copy number of plasmid introduced, it is clear that the presence of the heterologous partner is not an absolute prerequisite for the stable expression of these fusion proteins. These observations suggest that at least in certain cell types, the presence of both AP3 and PI proteins together is not necessary for protein stability. The cytoplasmic localization of the AP3 and PI fusion proteins in cells in which they are individually expressed and stable indicates that an AP3-PI interaction is required for the appropriate subcellular localization of these transcription factors.

\section{The role of nuclear colocalization in floral development}

DNA-binding activity has been demonstrated in vitro for the Antirrhinum DEF and GLO proteins, but only when both proteins are present, suggesting that DEF and GLO form heterodimers that bind to DNA (Schwarz-Sommer et al. 1992; Trobner et al. 1992; Zachgo et al. 1995). Based on the similarities between the homologous Arabidopsis and Antirrhinum proteins, it has been proposed that AP3 and PI also bind to DNA as a heterodimeric complex (Goto and Meyerowitz 1994; Jack et al. 1994). If this hypothesis is true, then nuclear colocalization would act as an additional mechanism to ensure the coordinated presence of the AP3 and PI proteins inside the nucleus. In addition, other factors as well as the AP3 and PI proteins appear to be required in the nucleus for floral development. When we expressed both the p(1-208)PIGUS and $p(1-232) A P 3-G U S$ constructs together in transgenic plants, we saw nuclear localization of GUS activity in a number of vegetative cell types but did not observe any corresponding obvious homeotic transformations. This observation suggests that other factors are required in conjunction with the nuclear-localized AP3 and PI proteins to effect appropriate floral development.

We have also observed that constructs containing AG translationally fused to GUS cannot be targeted to the nucleus in the onion cell transient assay system (data not shown). However, AG has been shown to bind to DNA in vitro, suggesting that it can act independently in transcriptional activation (Huang et al. 1993; Shiraishi et al. 1993). These observations suggest that other factors act in combination with AG to facilitate its entry into the nucleus but then may not be required to elicit AG transcriptional function. One possibility is that $A G$ function is first delimited by its spatial domain of transcriptional activation, and subsequently fine-tuned at the post-translational level at least in part by regulated nuclear localization. These observations suggest that nuclear colocalization may be a more generally utilized mechanism to ensure the coordinated function of floral homeotic gene products.

\section{Materials and methods}

Generation of constructs

Plasmid constructs were made using standard protocols (Sambrook et al. 1989). In general, for fusion proteins, various fragments were ligated into the expression vector pRTL2-GUS at appropriate restriction sites (Restropo et al. 1990). AP3 coding sequences and fragments were derived from the plasmid pIa (Irish and Yamamoto 1995); PI coding sequences and fragments from the plasmid pCPI-7Z, containing a full-length PI cDNA (Goto and Meyerowitz 1994); and AG sequences from pCIT 565 (Yanofsky et al. 1990). pSPI, pSAP3, and pSAGAM were generated by ligating either PI, AP3, or AG coding regions into the pRTL2-GUS/NIa vector (Restropo et al. 1990) from which the GUS and NIa sequences had been removed. The GUS (pRTL2GUS) and GUS-NIa (pRTL2-GUS/NIa) constructs were obtained from A. von Arnim (von Arnim and Deng 1994). Restriction and modification enzymes were purchased from New England Biolabs (Beverly, MA). Fusion junctions on all clones were verified by dideoxy sequencing of double-stranded DNA templates, using Sequenase (U.S. Biochemicals, Cleveland, OH). Details of individual plasmid constructions are available on request.

\section{Biolistic bombardment and transient assays}

DNA for bombardment was prepared using cesium chloride density gradients and quantitated spectrophotometrically. Epidermal peels from onion bulbs were placed on agar plates and subjected to bombardment with various plasmids according to the method of Varagona et al. (1992). The plates were then incubated in the dark for $24 \mathrm{hr}$ at $28^{\circ} \mathrm{C}$, and subsequently the 
onion epidermal peels were stained for $3 \mathrm{hr}$ at $37^{\circ} \mathrm{C}$ for $\beta$-glucuronidase activity in a solution of $100 \mathrm{~mm}$ sodium phosphate buffer ( $\mathrm{pH} 7.0), 1 \mathrm{~mm}$ EDTA (pH 8.0), 1\% Triton X-100, $5 \mathrm{~mm}$ potassium ferricyanide, $5 \mathrm{~mm}$ potassium ferrocyanide, and 2 $\mathrm{mg} / \mathrm{ml}$ of X-Gluc. Epidermal peels were mounted on microscope slides in $1 \mu \mathrm{g} / \mathrm{ml}$ of DAPI in $\mathrm{H}_{2} \mathrm{O}$. Slides were encoded and scored in a double blind fashion for nuclear versus cytoplasmic localization of GUS activity using bright-field optics, and the position of DAPI-stained nuclei was determined using epifluorescence. Data for each set of experiments were tabulated, and statistical analyses were performed using DeltaGraph (DeltaPoint, Inc., Monterey, CA) software to calculate the standard errors.

\section{Plant transformation and husbandry}

The fusion constructs under control of the cauliflower mosaic virus $35 \mathrm{~S}$ promoter were cloned into the binary plant transformation vector pBIN19 (Bevan 1984). These plasmids were transferred into Agrobacterium tumefaciens by electroporation (Shen and Forde 1989). Arabidopsis (Nossen wild-type ecotype) was transformed using Agrobacterium-mediated root transformation as described previously (Valvekens et al. 1988). Transformants were selected with $30 \mu \mathrm{g} / \mathrm{ml}$ of kanamycin. Over 40 independent transformant lines were obtained for each of the p(1-208)PI-GUS, p(1-232)AP3-GUS, and p(1-199)AP3-GUS constructs. Of $18 \mathrm{p}|1-208| \mathrm{PI}-\mathrm{GUS}$ lines tested, 16 showed GUS activity. Of $23 \mathrm{p}(1-232) \mathrm{AP} 3-\mathrm{GUS}$ lines examined, 22 showed detectable GUS activity. Of $11 \mathrm{p}(1-199)$ AP3-GUS lines examined, 9 showed GUS activity. For each construct, the transgenic lines showed similar patterns of GUS expression, although levels of GUS expression varied severalfold. Transgenic GUS and GUS-NIa seed were a gift of A. von Arnim (von Arnim and Deng 1994). Histochemical staining of GUS activity was performed by staining transgenic tissues under vaccuum for 30 min, followed by incubation in a modified GUS staining solution containing $1 \%$ Tween 20 instead of Triton X-100, at $37^{\circ} \mathrm{C}$ for $24 \mathrm{hr}$. Transgenic flowers to be analyzed were then fixed in FAA $(3.7 \%$ formaldehyde, $50 \%$ ethanol, $5 \%$ acetic acid $)$ and incubated in $70 \%$ ethanol overnight to remove the chlorophyll. Stained flowers were rehydrated and either whole mounted or individual organs were dissected out with glass needles and mounted in $1 \mu \mathrm{g} / \mathrm{ml}$ of DAPI in $100 \mathrm{~mm}$ phosphate buffer $/ \mathrm{pH}$ 7.0), and observed with bright-field optics and with epifluorescence. All plants were grown with a combination of fluorescent and incandescent light at $175 \mu$ moles $/ \mathrm{m}^{2}$ per sec under a $16-\mathrm{hr}$ day/8-hr night regime.

\section{Acknowledgments}

We thank Elliot Meyerowitz, Marty Yanofsky, and Albrecht von Arnim for gifts of plasmids and Elliot Meyerowitz for communication of results prior to publication. We thank Albrecht von Arnim, Christopher Day, Susan Carr, Theresa Hill, Elena Wright, Pablo Jenik, and Iain Dawson for helpful comments and advice during the course of this work. This research was supported by a Lavoisier fellowship from the French Ministère des Affaires Etrangères to K.B., and grants from the U.S. Department of Agriculture (9502665) and National Science Foundation (IBN-9220536) to V.F.I.

The publication costs of this article were defrayed in part by payment of page charges. This article must therefore be hereby marked "advertisement" in accordance with 18 USC section 1734 solely to indicate this fact.

\section{References}

Abel, S. and A. Theologis. 1995. A polymorphic bipartite motif signals nuclear targeting of early auxin-inducible proteins related to PS-IAA4 from pea. Plant J. 8: 87-96.

Bevan, M. 1984. Binary Agrobacterium vectors for plant transformation. Nucleic Acids Res. 12: 8711-8721.

Bowman, J.L., D.R. Smyth, and E.M. Meyerowitz. 1989. Genes directing flower development in Arabidopsis. Plant Cell 1: $37-52$.

- 1991. Genetic interactions among floral homeotic genes of Arabidopsis. Development 112: 1-20.

Bruhn, L. and G.F.J. Sprague. 1994. MCM1 point mutants deficient in expression of $\alpha$-specific genes: Residues important for interaction with $\alpha 1$. Mol. Cell. Biol. 14: 2534-2544.

Carrington, I.C., D.D. Freed, and A.J. Leinicke. 1991. Bipartite signal sequence mediates nuclear translocation of the plant potyviral NIa protein. Plant Cell 3: 953-962.

Dingwall, C. and R.A. Laskey. 1991. Nuclear targeting sequences-a consensus? Trends Biochem. Sci. 16: 478-481.

Fabre, E. and E.C. Hurt. 1994. Nuclear transport. Curr Opin. Cell Biol. 6: 335-342.

Fischer, U., J. Huber, W.C. Boelens, I.W. Mattaj, and R. Lührmann. 1995. The HIV-1 rev activation domain is a nuclear export signal that accesses an export pathway used by specific cellular RNAs. Cell 82: 475-483.

Garcia-Bustos, J., J. Heitman, and M.N. Hall. 1991. Nuclear protein localization. Biochem. Biophys. Acta 1071: 83-101.

Gauthier-Rouviere, C., M. Vandromme, N. Lautredou, Q.Q. Cai, F. Girard, A. Fernandez, and N. Lamb. 1995. The serum response factor nuclear localization signal: general implications for cyclic AMP-dependent protein kinase activity in control of nuclear translocation. Mol. Cell. Biol. 15: 433-444.

Goto, K. and E.M. Meyerowitz. 1994. Function and regulation of the Arabidopsis floral homeotic gene PISTILLATA. Genes \& Dev. 8: 1548-1560.

Harter, K., S. Kircher, H. Frohnmeyer, M. Krenz, F. Nagy, and E. Schafer. 1994. Light-regulated modification and nuclear translocation of cytosolic G-box binding factors in parsley. Plant Cell 6: 545-559.

Henkel, T., U. Zabel, K. van Zee, J.M. Muller, E. Fanning, and P.A. Baeuerle. 1992. Intramolecular masking of the nuclear location signal and dimerization domain in the precursor for the p50 NF- $\mathrm{B}$ B subunit. Cell 68: 1121-1133.

Hill, J.P. and E.M. Lord. 1989. Floral development in Arabidopsis thaliana: A comparison of the wild type and the homeotic pistillata mutant. Can. J. Bot. 67: 2922-2936.

Huang, H., Y. Mizukami, and H. Ma. 1993. Isolation and characterization of the binding sequence for the product of the Arabidopsis floral homeotic gene AGAMOUS. Nucleic Acids Res. 21: 4769-4776.

Huang, H., M. Tudor, Y. Zhang, Y. Hu, and H. Ma. 1996. DNA binding properties of two Arabidopsis MADS domain proteins: binding consensus and dimer formation. Plant Cell 8: 81-94.

Irish, V.F. and Y.T. Yamamoto. 1995. Conservation of floral homeotic gene function between Arabidopsis and Antirrhinum. Plant Cell 7: 1635-1644.

Jack, T., L.L. Brockman, and E.M. Meyerowitz. 1992. The homeotic gene APETALA3 of Arabidopsis thaliana encodes a MADS box and is expressed in petals and stamens. Cell 68: 683-697.

Jack, T., G.L. Fox, and E.M. Meyerowitz. 1994. Arabidopsis homeotic gene APETALA3 ectopic expression: Transcriptional and posttranscriptional regulation determine floral organ identity. Cell 76: 703-716. 
Klein, T.M., E.D. Wolf, R. Wu, and J.C. Sanford. 1987. Highvelocity microprojectile for delivering nucleic acids into living cells. Nature 327: 70-73.

Krizek, B.A. and E.M. Meyerowitz. 1996. The Arabidopsis homeotic genes APETALA3 and PISTILLATA are sufficient to provide the $\mathrm{B}$ class organ identity function. Development 122: 11-22.

Laskey, R.A. and C. Dingwall. 1993. Nuclear shuttling: The default pathway for nuclear proteins? Cell 74: 585-586.

Ma, H., M.F. Yanofsky, and E.M. Meyerowitz. 1991. AGLlAGL6, an Arabidopsis gene family with similarity to floral homeotic and transcription factor genes. Genes \& Dev. 5: 484-495.

Michael, W.M., M. Choi, and G. Dreyfuss. 1995. A nuclear export signal in hnRNP Al: A signal-mediated, temperaturedependent nuclear protein export pathway. Cell 83:415422.

Norman, A., M. Runswick, R. Pollock, and R. Treisman. 1988. Isolation and properties of cDNA clones encoding SRF, a transcription factor that binds to the c-fos serum resonse element. Cell 55: 989-1003.

Pellegrini, L., S. Tan, and T.J. Richmond. 1995. Structure of the serum response factor core bound to DNA. Nature 376: 490498.

Picard, D. and K.R. Yamamoto. 1987. Two signals mediate hormone-dependent nuclear localization of the glucocorticoid receptor. EMBO J. 6: 3333-3340.

Pnueli, L., M. Abu-Abeid, D. Zamir, W. Nacken, Z. SchwarzSommer, and E. Lifschitz. 1991. The MADS box gene family in tomato: temporal expression during floral development, conserved secondary structures and homology with homeotic genes from Antirrhinum and Arabidopsis. The Plant I. 1: 255-266.

Raikhel, N. 1992. Nuclear targeting in plants. Plant Physiol. 100: 1627-1632.

Rech, J., I. Barlat, J.L. Veyrune, A. Vie, and J.M. Blanchard. 1994. Nuclear import of serum response factor (SRF) requires a short amino-terminal nuclear localization sequence and is independent of the casein kinase II phosphorylation site. $J$. Cell Sci. 107: 3029-3036.

Restropo, M.A., D.D. Freed, and J.C. Carrington. 1990. Nuclear transport of plant potyviral proteins. Plant Cell 2: 987-998.

Richardson, W.D., A.D. Mills, S.M. Dilworth, R.A. Laskey, and C. Dingwall. 1988. Nuclear protein migration involves two steps: Rapid binding at the nuclear envelope followed by slower translocation through the nuclear pores. Cell 52: 655-664

Roth, S., D. Stein, and C. Nüsslein-Volhard. 1989. A gradient of nuclear localization of the dorsal protein determines dorsoventral pattern in the Drosophila embryo. Cell 59: 11891202.

Rushlow, C.A., K. Han, J.L. Manley, and M. Levine. 1989. The graded distribution of the dorsal morphogen is initiated by selective nuclear transport in Drosophila. Cell 59: 11651177.

Sambrook, J., E.F. Fritch, and T. Maniatis. 1989. Molecular cloning: A laboratory manual, 2nd ed. Cold Spring Harbor Laboratory Press, Cold Spring Harbor, NY.

Schmidt-Zachmann, M.S., C. Dargemont, L.C. Kuhn, and E.A. Nigg. 1993. Nuclear export of proteins: The role of nuclear retention. Cell 74: 493-504.

Schwarz-Sommer, Z., I. Hue, P. Huijser, P.J. Flor, R. Hansen, F. Tetens, W.-E. Lonnig, H. Saedler, and H. Sommer. 1992. Characterization of the Antirrhinum floral homeotic MADS-box gene deficiens: evidence for DNA binding and autoregulation of its persistent expression throughout flower development. EMBO I. 11: 251-263.

Shen, W. and B.G. Forde. 1989. Efficient transformation of Agrobacterium spp. by high voltage electroporation. Nucleic Acids Res. 17: 8385.

Shiraishi, H., K. Okada, and Y. Shimura. 1993. Nucleotide sequences recognized by the AGAMOUS MADS domain of Arabidopsis thaliana in vitro. Plant f. 4: 385-398.

Sommer, H., J.-P. Beltran, P. Huijser, H. Pape, W.-E. Lonnig, H. Saedler, and Z. Schwarz-Sommer. 1990. Deficiens, a homeotic gene involved in the control of flower morphogenesis in Antirrhinum majus: The protein shows homology to transcription factors. EMBO I. 9: 605-613.

Steward, R. 1989. Relocalization of the dorsal protein from the cytoplasm to the nucleus correlates with its function. Cell 59: 1179-1188.

Trobner, W., L. Ramirez, P. Motte, I. Hue, P. Huijser, W.-E. Lonnig, H. Saedler, H. Sommer, and Z. Schwarz-Sommer. 1992. Globosa-A homeotic gene which interacts with deficiens in the control of Antirrhinum floral organogenesis. EMBO 1. 11: 4693-4704.

Valvekens, D., M. Van Montagu, and M. Van Lijsebettens. 1988. Agrobacterium tumefasciens mediated transformation of Arabidopsis root explants by using kanamycin selection. Proc. Natl. Acad. Sci. 85: 5536-5540.

van der Krol, A.R. and N.-H. Chua. 1991. The basic domain of plant B-ZIP proteins facilitates import of a reporter protein into plant nuclei. Plant Cell 3: 667-675.

Varagona, M.J., R.J. Schmidt, and N.V. Raikhel. 1992. Nuclear localization signal(s) required for nuclear targeting of the maize regulatory protein opaque-2. Plant Cell 4: 1213-1227.

von Arnim, A.G. and X.-W. Deng. 1994. Light inactivation of Arabidopsis photomorphogenetic repressor COP1 involves a cell-specific regulation of its nucleocytoplasmic partitioning. Cell 79: 1035-1045.

Weigel, D. and E.M. Meyerowitz. 1994. The ABCs of floral homeotic genes. Cell 78: 203-209.

Wen, W., J.L. Meinkoth, R.Y. Tsien, and S.S. Taylor. 1995. Identification of a signal for rapid export of proteins from the nucleus. Cell 82: 463-473.

Yanofsky, M.F., H. Ma, J.L. Bowman, G.N. Drews, K.A. Feldmann, and E.M. Meyerowitz. 1990. The protein encoded by the Arabidopsis homeotic gene agamous resembles transcription factors. Nature 346: 35-39.

Zachgo, S., E. de Andrade Silva, P. Motte, W. Trobner, H. Saedler, and Z. Schwarz-Sommer. 1995. Functional analysis of the Antirrhinum floral homeotic Deficiens gene in vivo and in vitro by using a temperature-sensitive mutant. Development 121: 2861-2875. 


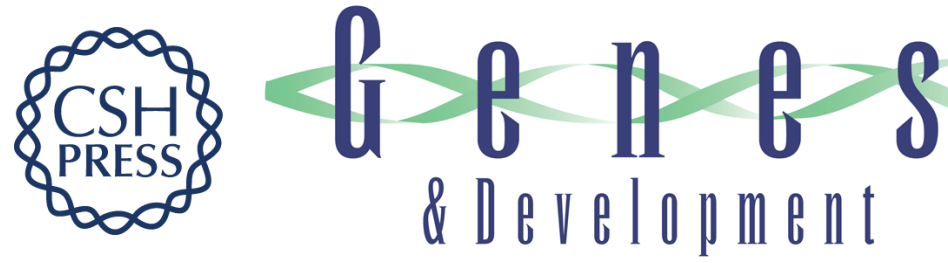

\section{Nuclear localization of the Arabidopsis APETALA3 and PISTILLATA homeotic gene products depends on their simultaneous expression.}

B McGonigle, K Bouhidel and V F Irish

Genes Dev. 1996, 10:

Access the most recent version at doi:10.1101/gad.10.14.1812

References This article cites 50 articles, 18 of which can be accessed free at:

http://genesdev.cshlp.org/content/10/14/1812.full.html\#ref-list-1

License

Email Alerting

Service

Receive free email alerts when new articles cite this article - sign up in the box at the top right corner of the article or click here.

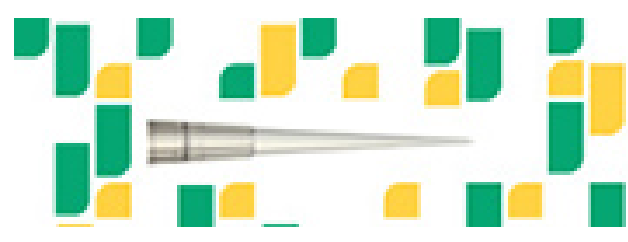

Focused on your science. 